Infrared Absorption Spectrum of Water adsorbed on $\alpha$-Helical Synthetic Polypeptides

I REPORT here some observations on the polarized infrared absorption spectrum of water adsorbed on orientated synthetic polypeptide films. The results suggest that the water molecules are adsorbed at specific sites and orientations with respect to the substrate molecules.

Orientated films of high molecular weight synthetic polypeptides in the $\alpha$-helical conformation were prepared by collapsing to one end of a Langmuir trough monolayers spread on the water surface. The method used to spread the monolayers and to remove the collapsed film has been described previously ${ }^{1,2}$. Sufficient monolayers wero spread to give a specimen with an absorbance of 1.5 or yreater for the amide I band. Films were mounted on harium fluoride plates and dried to a predetermined humidity. Where necessary, scatter of radiation from the surface of the specimen was reduced by allowing a drop of chloroform or benzene to flow across the surface so that it softened and became compacted. This treatment improved the quality of the spectra and probably modified the crystallinity of the specimen, but was not essential to see the main features described. Because the $\mathrm{OH}$ stretching band lies close to the amide A (NH-stretching) band, it is helpful (but not essential) to reduce the NH absorption by using $\mathrm{N}$-deuterated polymer and spreading the monolayers on $0.01 \mathrm{M} \mathrm{HCl}$ on which the back exchange of deuterium is slow ${ }^{1}$.

High molecular weight specimens of poly-D-alanine, poly- $\gamma$-ethyl-L-glutamate and poly- $\gamma$-methyl-L-glutamate have been examined with particular reference to the $2,000-4,000 \mathrm{~cm}^{-1}$ spectral range. Although the strength and shape of the water absorption band varies from one polymer to another, three main features are common to all the polymers: (I) the OH absorption is not centred about $3,400 \mathrm{~cm}^{-1}$ as in liquid water, but is displaced to around $3,500 \mathrm{~cm}^{-1}$; (2) the band is clearly made of two or three components; (3) overall, the band exhibits marked perpendicular dichroism. Fig. 1 illustrates these features for poly- $\gamma$-methyl-L-glutamate, together with the $\mathrm{NH}$ and $\mathrm{N}^{2} \mathrm{H}$ stretching bands (about 3,300 and $2.450 \mathrm{~cm}^{-1}$ ), which

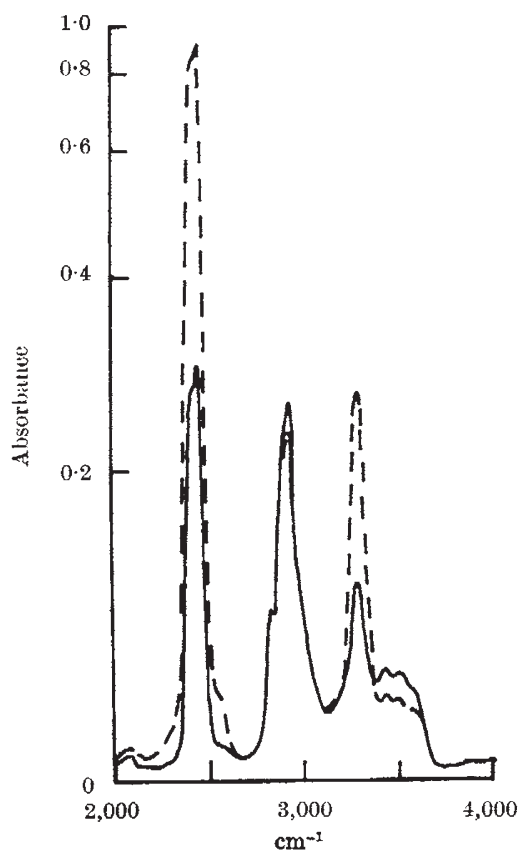

Fig. 1. Infrared absorption spectrum of a partially $\mathrm{N}$-deuterated flim of noly- $\gamma$-methyl-I-glutamate, $30^{\circ} \mathrm{C}$, approximately 91 per cent relative humidity. Full line: electric vector perpendicular to di
orientation; broken line: electric vector parallel. are of opposite dichroic character to the water band, and the $\mathrm{CH}$ bands. The water band has components at about $3,440,3,510 \mathrm{~cm}^{-1}$ and $3,570 \mathrm{~cm}^{-1}$. Poly- $\gamma$-ethyl-L-glutamate has three similar bands and poly-D-alanine has a band at about $3,470 \mathrm{~cm}^{-1}$ with a shoulder at $3,530 \mathrm{~cm}^{-1}$.

Because the frequency of the water absorption band is roughly mid-way between that of the free and hydrogenbonded $\mathrm{OH}$ stretching frequencios it is probable that the hydrogen bonding component of the binding is weak. Tho dichroism and splitting of the band into two or three components is good evidence that the molecules are in specific orientations and sites with respect to the polymer. If the band is attributed mainly to the $\mathrm{OH}$ antisymmetrical stretching vibration (normally the strongest), which has a transition moment parallel to the line drawn through the hydrogen atoms, the molecules are orientated on average with this direction fairly close to planes drawn perpendicular to the helix axis. An estimate of the angle can be made by using the direction of the $\mathrm{NH}$ and $\mathrm{N}^{2} \mathrm{H}$ transition moments to give a measure of the orientation of the polymer. The usual model for fibre orientation is assumed with a fraction of perfectly orientated polymer and the rest disorientated ${ }^{3}$, and the water is taken to be distributed in proportion over both fractions. This leads to the $\mathrm{H}-\mathrm{H}$ direction being within about $25^{\circ}$ to the plane perpendicular to the axis of the helix.

Weak perpendicular dichroism of the same water band has been observed in hydrated $\alpha$-keratin by- Bendit 4 , who found the frequency to be $30.40 \mathrm{~cm}^{-1}$ higher than in liquid water. Although the spectrum was very similar to that of an equivalent thickness of liquid water, it is probable that the observations are closely related to the present work.

A tentative explanation of these observations, consistent with the views of Bendit for keratin, is that the water molecules orientate with a hydrogen atom directed towards the peptide oxygen, possibly forming a weak hydrogen bond to it, with the $\mathrm{H}-\mathrm{H}$ direction of the water molecule within $25^{\circ}$ to the plane perpendicular to the helix axis. In this situation it is probable that the water dipole will interact strongly with the peptide dipole. This orientation would account for the positive sign of the surface potential when a monolayer of $\alpha$-helices is spread on water ${ }^{1}$, if on a clean water surface the hydrogen atoms aro normally directed downwards ${ }^{5}$. In the case of the glutamate polymers an additional interaction may nccur between the water and the side chain carbonyl.

This work is supported by the Science Research Council and I thank Miss L. Mallaby for technical assistance.

\section{B. R. MALCOLMI}

Department of Molecular Biology,

University of Edinburgh,

Edinburgh EH9 3JR.

Received February 5, 1970.

${ }^{1}$ Mralcolm, B. R., Proc. Roy. Soc., A, 305, 363 (1968).

"Valcolm, B. R., Biochem. J., 110, 733 (1968).

${ }^{3}$ Elliott, A., Infrared Spectra and Structure of Organic Long-chain Polymers, 66 (Edward Arnold, 1969).

4 Bendit, E. G., Biopolymers, 4, 539 (1966).

${ }^{5}$ Bernal, J. D, in The State and Mocement of Water in Living Organisms

\section{Involvement of Articular Cartilage in a Linear Relaxation Process during Walking}

A KNEE joint consists essentially of a pair of articulating bone surfaces lined with cartilage; the latter is swollen with an aqueous solution of protein and salts and contained within a sac (synovial membranc). The solution to prob- 